\title{
Implementation intentions facilitate prospective memory under high attention demands
}

\author{
Mark A. McDaniel and Daniel C. Howard \\ Washington University, St. Louis, Missouri \\ AND \\ KARIN M. BUTLER \\ University of New Mexico, Albuquerque, New Mexico
}

\begin{abstract}
An implementation intention is a planning technique that involves specifying a situation for initiating an intended action and linking these specific cues to the intention. In two experiments with young adults, we found significant increases in prospective memory with implementation intentions. With an implementation intention, but not with standard instructions, prospective memory performance was maintained under demanding attentional conditions (Experiment 2). Ongoing task performance did not decline, however, in relation with a no prospective memory control. Positive effects were not observed when the imagery component of the implementation intention was isolated from the verbal component. We suggest that implementation intention planning (relative to standard instructions) increases the likelihood that people will encode a robust associative link between the target cue and the intended action, thereby promoting reflexive triggering of the intended action on presentation of the target cue.
\end{abstract}

A hallmark of human behavior is the formation of intentions that specify actions to be carried out in the future. This behavior is reflected in the common New Year's ritual of forming resolutions for positive actions that an individual wants to initiate during the following year. More generally, people from time to time form intentions that bear on personal health, such as the intention to exercise more often or to take a vitamin supplement. The formation of good intentions, however, does not necessarily ensure successful execution of these intentions. As Gollwitzer (1999) humorously noted, folklore indicates that "the road to hell is paved with good intentions" (p. 493). To improve execution of intended goals and behaviors, Gollwitzer developed a planning technique that involves (1) clearly specifying the situation (where and when) that will be a good opportunity for initiating the intended action and (2) linking these specific situational cues to the intended action. This technique results in an implementation intention.

Much research has demonstrated that forming implementation intentions can significantly increase the likelihood that an intention will be executed. For instance, Sheeran and Orbell (1999) found that participants instructed to form implementation intentions for remembering to take a daily vitamin $\mathrm{C}$ tablet over the course of 3 weeks (participants had to plan where and when they would take the tablet each day) remembered to take the vitamin $\mathrm{C}$ tablet more often than did participants who formed the general intention of taking a vitamin C tablet each day (see also Gollwitzer, 1999; Liu \& Park, 2004; Milne, Orbell, \& Sheeran, 2002; Orbell, Hodg- kins, \& Sheeran, 1997). A number of studies document that implementation intentions are effective, but several key issues remain unresolved. First, uncertainty persists regarding possible boundary conditions of implementation intention effects. Second, and in a related vein, little is known about the particular components of an implementation intention that support its effectiveness or about the mechanisms by which implementation intentions produce their positive effects. The present study bears on these general issues.

\section{Implementation Intentions and Automatic Processes}

Gollwitzer (1999) contended that encoding an intended action as an implementation intention has several cognitive consequences, with a primary consequence being that the linkage of the intended action to specific situational cues allows automatic triggering of the intention when the cues are encountered. Automatic triggering of the intention would obviate the need for attentional resources that might otherwise be required in order to support retrieval of the intention, such as resources needed to self-initiate retrieval of the intention (Craik, 1986), to monitor the environment for an appropriate moment for performing the intention (Smith, 2003), or to maintain the intention in a heightened state of activation so that it would readily come to mind (Guynn, 2003). Thus, the idea is that implementation intentions increase execution of the intended behavior by reducing reliance on resource-demanding processes and fostering relatively automatic retrieval.

M.A. McDaniel, mmcdanie@artsci.wustl.edu 
One implication of this idea is that the positive effects of implementation intentions should prevail, even when cognitive resources are limited. This expectation was confirmed in two studies that found that older adults, who are assumed to have generally reduced cognitive resources (Craik \& Byrd, 1982) in an implementation intention condition, outperformed older adults in a control condition without implementation intentions (Chasteen, Park, \& Schwarz, 2001; Liu \& Park, 2004). However, positive implementation intention effects with older adults provide only indirect support for the automatic retrieval hypothesis. It is possible that, for some reason, the implementation intention groups adopted resource allocation policies that favored execution of the intended action, possibly at the expense of ongoing task performance.

To evaluate the preceding possibility, Cohen and Gollwitzer (2008) examined implementation intentions in the context of a laboratory prospective memory task. Prospective memory tasks typically involve the challenge of remembering to perform intended actions in the future when one is busily engaged in an ongoing activity that distracts the person from the intended activity (see McDaniel \& Einstein, 2007a, for a more extensive delineation of prospective memory tasks). The novel aspect of Cohen and Gollwitzer's approach was their evaluation of the extent to which adopting a prospective memory task interfered with performance of the ongoing activity when implementation intentions were formed (at the time of encoding). Specifically, response times for performing the ongoing activity alone (a lexical decision task) and for performing the ongoing activity in the presence of a prospective memory task were compared. Cohen and Gollwitzer found that (1) with implementation intentions, the prospective memory task did not exact a cost from the ongoing activity; however, costs were evident under standard prospective memory instructions; and (2) implementation intentions increased levels of prospective remembering relative to standard instructions. These patterns imply that implementation intentions fostered relatively automatic retrieval.

In the present study, we adopted an approach that was complementary to that of Cohen and Gollwitzer (2008) in order to examine the implementation intention effect. We directly limited the resource availability for the prospective memory task by increasing the attentional demands of the ongoing activity in some portions of the experiment (by requiring participants to perform a secondary task in addition to the ongoing activity). We reasoned that performing the ongoing activity and the secondary task conjointly would severely limit resources available to support prospective remembering (i.e., noticing the appropriate moment for the prospective memory activity and retrieval of the prospective memory intention), thereby reducing prospective memory performance in the no implementation intention control (see Marsh \& Hicks, 1998; McDaniel, Guynn, Einstein, \& Breneiser, 2004). To the extent that implementation intentions support relatively automatic triggering of the intended action on presentation of the target cue (a particular word in this case), prospective memory should not be impaired in the presence of the secondary task in the implementation intention condition. In an initial study, Brandstätter, Lengfelder, and Gollwitzer (2001) reported this pattern, but there was ambiguity regarding whether their high-attention-demand conditions significantly increased resource demands, and there was no inclusion of a no prospective memory control condition to assess for possible resource allocation policies that disfavored ongoing task performance to maintain prospective memory performance.

A second general issue addressed by the present study is whether implementation intentions prove effective in enhancing prospective memory as typically instantiated in the laboratory and with young adults. This is an interesting question because the elements of the standard prospective memory task bear strong similarity to the fundamental elements of implementation intentions (see Ellis \& Freeman, 2008). For instance, in many prospective memory experiments, including the present ones, the prospective memory task is performed in the context of an ongoing activity (e.g., word rating task), and participants are told to try to remember to press a particular key on the keyboard if a certain target (e.g., word) should appear. In this case, the prospective memory task specifies a retrieval cue (word) processed fully in service of the ongoing activity (e.g., rating features of individual words) and specifies a particular action to be executed when the cue appears (e.g., press the "Q" key). Similarly, with an implementation intention, a person expresses that he or she will take a particular action when a particular situation (retrieval cue) occurs. ${ }^{1}$

Given these similarities between the features of laboratory prospective memory instructions and implementation intentions, it is not certain that implementation intentions should promote higher levels of prospective memory performance than the standard prospective memory instructions, at least for young adults. In line with this possibility, in several unpublished experiments, researchers have failed to find any hint of an implementation intention effect in a standard prospective memory paradigm (Bennett, Clawson, \& Kardiasmenos, 2005; Kardiasmenos, Clawson, Wilken, \& Wallin, 2004), and in a published experiment no implementation intention effect was reported in a modified prospective memory paradigm (Einstein, McDaniel, Williford, Pagan, \& Dismukes, 2003).

Alternatively, there is an important difference between the standard prospective memory instructions and implementation intentions that might change performance levels across these conditions. The implementation intention forces participants to focus encoding on a particular retrieval target and to form an associative link between that target and an intended activity (as detailed in the following Method section). In contrast, the standard instruction condition provides less guidance in terms of intention encoding, thereby leaving open the possibility that at least some participants will not spontaneously form an especially good encoding of the target event and its association to the intended action. Supporting this possibility, Kliegel, Martin, McDaniel, Einstein, and Moor (2007) analyzed participants' overtly constructed plans for executing a prospective memory task and found that only some of the plans contained elements that were analogous to implementation-intention-type planning. Accordingly, implementation intentions may force effective encoding 
of the intention for a greater proportion of participants than would otherwise engage (spontaneously) such encoding under standard prospective memory instructions. Cohen and Gollwitzer's (2008) finding (described above) of a significant implementation intention effect is consistent with this possibility. In the present experiments, we provide further investigation of whether implementation intentions enhance performance for young adults in laboratory prospective memory tasks.

\section{EXPERIMENT 1}

The components of an implementation intention instruction have varied across experiments, so there is no standard technique by which implementation intentions are instantiated in the laboratory. In Cohen and Gollwitzer (2008), the implementation intention condition required participants to write down three times the implementation intention. Chasteen et al. (2001) similarly required participants to echo an implementation intention phrase, but bolstered the phrase by a 30 -sec interval in which participants were instructed to imagine themselves performing the intended action on seeing the target cue. In the present experiment, we followed the Chasteen et al. technique of including visual imagery in the implementation intention.

\section{Method}

Design and Participants. The design was a $2 \times 2$ mixed factorial with type of prospective memory instruction (no implementation intention control, implementation intention) as the betweenparticipants factor and attentional demand (standard, high demand) as the within-participants factor. The participants were 34 undergraduates enrolled in psychology courses at the University of New Mexico, who participated in partial fulfillment of a course requirement. There were 16 participants in the control instruction group and 18 in the implementation intention group.

Materials. There were two prospective memory target words ( $s p a-$ ghetti, doll). The remaining 232 nontarget words were selected from the MRC Psycholinguistic Database (Version 2.0) and ranged from 232 to 634 in concreteness, from 290 to 643 in imaginability, from 258 to 639 in familiarity, and from 1 to 193 in frequency. Altogether, the words were presented in four 60 -word blocks. The prospective memory target words were presented in each block on either the 25th or 26 th trial and on either the $51 \mathrm{st}$ or 52 nd trial. All other trials contained nontarget words and were presented in random order.

The secondary task was an odd-digit detection task, in which digits (1-9) were presented every $2 \mathrm{sec}$ auditorily. Participants were instructed to press a button on a counter every time they heard two odd digits in a row. The digit-detection task was interleaved with periods of silence when the secondary task was not performed; these intervals varied from 30 to $60 \mathrm{sec}$. In total, there were 53 instances of two odd digits in a row. Prospective memory target words were always presented at least $15 \mathrm{sec}$ after the beginning or completion of a secondary task interval. The interleaving of the secondary task and periods of silence resulted in a divided-attention situation during half of the word rating task and a nondivided-attention situation during the other half of the task. The placement of the odd-digit detection periods was such that each participant encountered four of the prospective memory target words under divided attention and four of the prospective memory target words under nondivided attention. Each participant encountered either the first four or last four prospective memory targets under divided attention, counterbalanced by condition.

As a delay task between the prospective memory instructions and prospective memory task, participants completed the operation span task (OSPAN; Turner \& Engle, 1989). In this task, each dis- play contains a math equation followed by a word-for example, IS $(2 \times 1)+1=3$ ? CAT. Participants were instructed to read the equation aloud, to verify whether the equation was correct by saying "yes" or "no," and then to say the word aloud and remember it for a memory test. Following a set of two to six equation-word displays, participants were asked to recall the words in the order they were presented. Three trials of each set size were presented.

Procedure. The experiment lasted approximately $1 \mathrm{~h}$. Participants were told that we would be testing their perceptions of words, while at the same time, they would be doing a listening task. The listening task was the odd-digit detection task; these secondary instructions were given first. Participants were given a hand counter and asked to hold it in their nondominant hand. They were told that they would be listening to a series of numbers and that they should press the button on the counter when they heard two odd digits in a row. They were then given $30 \mathrm{sec}$ to practice the odd-digit detection task. Next, participants were informed that they would be rating words on a number of different dimensions. The word to be rated and the dimension on which the word was to be rated (familiarity, pleasantness, vividness, imagery) were presented on the screen for $7 \mathrm{sec}$. The participants rated the word from 1 (not at all) to 5 (very) by pressing the appropriate key on the number pad. Participants completed four practice trials for this task, with each trial containing a different word and one of the four dimensions.

After the practice trials, participants were presented with the prospective memory instructions. The instructions were presented in three phases: prospective memory statement, imagining, and repetition of instructions. In both conditions, participants silently read the prospective memory statement for the prospective memory task (If you see the words "spaghetti" or "doll" during the word rating task, press the " $Q$ " key). In the imagining phase, all participants were asked to imagine the event occurring and imagine themselves completing the task (Imagine the word "spaghetti" coming onto the screen and imagine yourself pressing the " $Q$ " key in response to it. Do the same for the word "doll"). Following Chasteen et al. (2001), participants were given $30 \mathrm{sec}$ to imagine themselves performing the task and were instructed to use the entire $30 \mathrm{sec}$. The difference between the control and implementation intention conditions was how the participants were asked to repeat the instructions after the imagining phase. In the control condition, participants were asked to restate the prospective memory instructions (Please tell the experimenter what you intend to do), consistent with typical prospective memory paradigms. Participants were not allowed to continue with the experiment until they were able to recall both prospective memory cue words and the response to be made to them. In the implementation intention condition, participants were given the implementation intention and asked to read it from the screen (e.g., Please tell the experimenter, "When I see the word 'spaghetti,'I press the ' $Q$ ' $k e y$, and when I see the word 'doll,'I press the 'Q'key').

After receiving the prospective memory instructions, participants answered questions about their age, sex, years of education, and general health status. Then they completed the OSPAN task. These delay tasks took approximately $15 \mathrm{~min}$ to complete. Then participants were reminded of the instructions for the word rating task and the odd-digit detection task and informed that they would be doing these tasks next. They were not reminded of the prospective memory instructions. The word rating and odd-digit detection tasks took about 30 min to complete. On completing the word rating task, participants were given a retrospective memory test for the prospective memory targets and intended actions for both prospective memory tasks.

\section{Results and Discussion}

For each participant, we computed the proportion of trials for which the prospective memory action was performed (pressing the "Q" key) under the standard attentional demands and under high attention demands. These data were submitted to a mixed $2 \times 2$ ANOVA. The alpha level for concluding significance was set at .05, and partial 
eta-squared $\left(\eta_{\mathrm{p}}^{2}\right)$ was computed as an index of effect size. Implementation intentions significantly increased prospective memory performance $(M=.92)$ relative to the control condition, not instructed to form an implementation intention $(M=.64)\left[F(1,31)=4.68, M S_{\mathrm{e}}=.26, \eta_{\mathrm{p}}^{2}=.13\right]$. The implementation intention effect held for both the standard attention condition $(M \mathrm{~s}=.90 \mathrm{vs} . .67)$ and the demanding attention condition $(M \mathrm{~s}=.93$ vs. .62) $[F(1,32)=1.49$, for the interaction]. However, the demanding attention condition (inclusion of a secondary task) did not attenuate prospective memory performance $(F<1)$.

This result, along with the recent finding by Cohen and Gollwitzer (2008), establishes that implementation intentions can significantly benefit prospective memory performance in young adults. The effect is likely not an artifact of differential cognitive capacity of the participants across conditions. Working memory capacity, as measured by the OSPAN task, did not significantly differ across instructional conditions $(M=15.1$ in the control instruction condition, and $M=17.2$ in the implementation intention condition; $F<1$ ).

Finding an implementation intention effect in the present prospective memory paradigm (and also in Cohen \& Gollwitzer, 2008) provides some insight into the spontaneous encoding/planning processes engaged when participants are faced with a prospective memory task. In the control condition, a particular target event and concrete intended action were specified for participants - elements also included in an implementation intention. The control condition, however, received less guidance than the implementation intention condition on linking the target event and intended action. Because of the less explicit guidance, at least some participants in the control condition presumably did not forge an adequate link between the given target event and the intended action, whereas others may have spontaneously done so (see Kliegel et al., 2007 , for an examination of overt prospective memory plans that accords with this possibility). Consistent with this analysis, in the control condition, 5 of the participants failed to respond on any of the prospective memory trials, whereas half of the participants (8) responded on every prospective memory trial. By contrast, in the implementation intention condition, 1 participant failed to respond on any prospective memory trial, and three quarters (14) responded on every trial.

Furthermore, for participants in the control condition who do sufficiently encode the target event-action link, prospective remembering may not necessarily rely on resource demanding processes (for the type of prospective remembering task used herein in which a specified target event is processed as part of the ongoing activity; for detailed development of this perspective see Einstein et al., 2005; McDaniel \& Einstein, 2000, 2007b). This possibility is consistent with the absence of divided attention effects in the control condition. The idea here is that automatic processing (for prospective memory) presumed to be supported by implementation intentions is essentially stimulated in normal prospective memory tasks when the participants adequately encode the particular target cuespecific action association during standard prospective memory instructions (see, e.g., Cohen, Jaudas, \& Gollwitzer, 2008).

Alternatively, the overall absence of divided attention effects may have been because the digit monitoring task was not demanding enough, especially in conjunction with the 7 -sec presentation rate for the ongoing word rating task, to limit resources sufficiently to produce prospective memory decrements (though McDaniel et al., 2004, and McDaniel, Robinson-Riegler, \& Einstein, 1998, reported prospective memory decline with a similar secondary task). Accordingly, Experiment 2 was conducted to remedy these potential procedural problems. We replaced digit monitoring with a secondary task used by Marsh and Hicks (1998) that places high demands on executive/ attentional resources, and we increased the presentation rate for the word rating task.

\section{EXPERIMENT 2}

Marsh and Hicks (1998) found that prospective memory performance was significantly impaired when random number generation (RNG) was performed, in comparison with standard attention conditions. Thus, in this experiment, we used RNG as a secondary task to the ongoing activity in order to create high demands on central executive (attentional) resources. Accordingly, we expected in this high-demand condition that with standard prospective memory instructions, prospective memory would decline. Of central interest was whether implementation intention encoding would prevent prospective memory decline in the face of these high attentional demands.

To gauge the extent to which intact prospective memory performance under high attentional demands (if found) was a consequence of relatively automatic processes promoted by implementation intention usage, we included a condition that required performing only the ongoing tasks, with no prospective memory obligations. We reasoned that if prospective memory performance was maintained under high attentional demands by allocating fewer resources to the ongoing task, then performance on the ongoing task would suffer in the prospective memory conditions relative to that of the no prospective memory controls.

Another issue examined in this experiment concerned the degree to which imagery encoding alone might produce gains in prospective memory (in relation to standard prospective memory instructions, in which participants are not required to imagine themselves performing the intended action), as well as possible immunity to high attentional demands. Past research that has focused on applying implementation intentions to prospective memory has not attempted to isolate the imagery encoding component from the if . . then statement (Chasteen et al., 2001; Liu \& Park, 2004). From a theoretical perspective, it is of interest to gain some understanding of the functional components of the implementation intention. Accordingly, we added a standard instructional group that encoded the prospective memory intention by simply reading and repeating the prospective memory instruction, and also retained the two instructional groups from Experiment 1. The new readonly group provided a control against which to evaluate 
effects produced by the imagery component alone (i.e., the control in the previous experiment).

Einstein et al. (2003, Experiment 3) reported that an implementation intention instantiated solely in terms of imagery encoding produced no gains in prospective memory performance relative to a typical verbal encoding instruction. However, their procedure limited participants to $6 \mathrm{sec}$ for imagery encoding. One might argue that more time is required for imagery encoding to be effective. Therefore, in the present experiment, we provided a generous $30 \mathrm{sec}$ for imagery encoding.

\section{Method}

Design and Participants. The design was a $3 \times 2$ mixed factorial design with type of prospective memory instruction (read-only, imagery, or implementation intention) as the between-participants factor and attentional demand (standard or high demand) as the within-participants factor. A no prospective memory control group was also tested to gauge baseline RNG and ongoing task performance in the absence of a prospective memory task. The participants were 130 undergraduates enrolled in psychology courses at Washington University in St. Louis who participated in partial fulfillment of a course requirement or for a small payment. Two participants were tested but excluded from the analysis due to a failure to remember the prospective memory task on a postexperimental retrospective memory test for the task. The remaining 128 participants were equally divided among the four groups (32 per group).

Procedure. The experiment lasted approximately $1 \mathrm{~h}$. Participants were first told that they would be tested on their ability to generate random numbers. Participants read a page of instructions detailing how to generate a good random number sequence. They were instructed to use the numbers 1 through 10, inclusive, to try to use each number as often as the other numbers, and to avoid any conscious patterns. Participants were informed that they would be saying the random numbers aloud, in time with a metronome that was set at one beat per second. They were then given $30 \mathrm{sec}$ to practice the RNG task. Next, participants were informed that they would be rating words on a number of different dimensions. This task was identical to that in Experiment 1, with the exception that the presentation rate was increased to $5 \mathrm{sec}$ per word, and the number of nontarget rating trials was increased from 232 to 328 , allowing 80 nontarget trials for each of four blocks, with the prospective memory targets placed at the 36th and 72nd trial of each block.

After four practice rating trials, participants in the three prospective memory conditions were presented with the prospective memory instructions. The instructions were presented in one of three formats: read-only, imagery, and implementation intention. In the read-only condition, participants read the prospective memory instructions for the primary prospective memory task (If you see the words "spaghetti" or "doll" during the word rating task, press the " $Q$ " key) and then repeated the instructions back to the experimenter. In the imagery condition, participants read the same prospective memory instructions and were then instructed to imagine themselves completing the task in the future. They were given $30 \mathrm{sec}$ to complete this imagery task and were instructed to use the entire $30 \mathrm{sec}$. At the end of the $30 \mathrm{sec}$, participants repeated the prospective memory instructions back to the experimenter. In the implementation intention condition, participants read the same prospective memory instructions and then completed the same imagery task as did the participants in the imagery condition. Participants then read the following statement aloud: "When I see the word spaghetti, I will press the 'Q' key, and when I see the word doll, I will press the 'Q' key." Control participants who did not perform the prospective memory task were not given the above instructions.

After receiving the prospective memory instructions, participants engaged in a distractor task, in which they completed arithmetic problems presented on a computer screen for $3 \mathrm{~min}$. After completing this task, participants were reminded of the instructions for the word rating task and were informed that they would be doing that task next. They were not reminded of the prospective memory instructions. Participants were also informed that during the word rating task, they would be prompted periodically to generate random number sequences aloud, and that they should continue to generate the number sequences until prompted to stop. Participants were instructed to continue performing the word rating task at the same time. In total, participants were prompted to generate random number sequences 20 times, with each generation period lasting between 30 and $55 \mathrm{sec}$. This resulted in a divided-attention situation during half of the word rating task and a nondivided-attention situation during the other half of the task. The placement of the number generation periods was such that each participant encountered four of the prospective memory target words under divided attention and four of the prospective memory target words under nondivided attention. Each participant encountered either the first four or last four prospective memory targets under divided attention, counterbalanced by condition.

Upon completing the word rating task, participants were given a stapled, three-page copy of Kuhl's (1994) ACS-90 personality inventory to fill out. On completing the inventory, participants were given a retrospective memory test for the prospective memory targets and intended actions for the prospective memory task.

\section{Results and Discussion}

Prospective memory performance. A prospective memory response was considered correct if the participant pressed the "Q" key while the word was presented on the screen; all prospective memory responses occurred during the presentation of the target word. Table 1 presents the mean performances as a function of instructional condition and attentional demands. These data were submitted to a mixed $3 \times 2$ ANOVA, with instruction condition (read-only, imagery, or implementation intention) as the between-participants factor and attentional demand (standard or high demand) as the within-participants factor.

There was a marginal main effect of instruction condition $\left[F(2,93)=3.52, M S_{\mathrm{e}}=3.65, p<.10, \eta_{\mathrm{p}}^{2}=.05\right]$. Planned comparisons revealed that participants in the implementation intention condition $(M=.95, S D=.11)$ performed marginally better than did participants in the read-only condition $(M=.89, S D=.18)[F(1,62)=3.65$, $\left.M S_{\mathrm{e}}=3.52, p<.10, \eta_{\mathrm{p}}^{2}=.06\right]$ and significantly better than participants in the imagery-only condition $(M=.87$, $S D=.24)\left[F(1,62)=4.82, M S_{\mathrm{e}}=7.56, p<.05, \eta_{\mathrm{p}}^{2}=\right.$ $.07]$. This result reinforces the conclusion from Experiment 1 , that implementation intentions can produce gains in prospective memory in younger adults. The new finding here is that $30 \mathrm{sec}$ of imagining oneself performing the action in the presence of the prospective memory cue (the imagery-only encoding groups) were not sufficient to produce any increase in prospective memory, in comparison with the read-only group.

Table 1

Mean Proportions of Prospective Memory Responses, As a Function of Instructional Group and Attention Demands, in Experiment 2

\begin{tabular}{lccc}
\hline & \multicolumn{2}{c}{ Attention Demands } & \\
\cline { 2 - 3 } \multicolumn{1}{c}{ Instructional Group } & Standard & High & Mean \\
\hline Implementation intention & .95 & .95 & .95 \\
Imagery only & .91 & .82 & .87 \\
Read only & .94 & .84 & .89 \\
\hline
\end{tabular}


A second critical finding was that prospective memory performance generally declined in the high-attentiondemand condition $(M=.87, S D=.22)$ in relation to the standard attention condition $(M=.94, S D=.16)$ $\left[F(1,93)=9.58, M S_{\mathrm{e}}=3.26, p<.01, \eta_{\mathrm{p}}^{2}=.09\right]$. This effect replicated Marsh and Hicks (1998; see also McDaniel et al., 1998, Experiment 3). More importantly, this effect allowed examination of whether implementation intentions would prevent prospective memory decline under high attention demands. A marginally significant interaction between attentional demand and instruction condition $\left[F(2,93)=2.41, M S_{\mathrm{e}}=3.26, p<.10, \eta_{\mathrm{p}}^{2}=.05\right]$ suggested that the decrements in prospective memory under high attention demands were limited to the groups not instructed with implementation intentions. Planned comparisons confirmed that performance was significantly lower for high-attention-demand trials in comparison with the standard attention trials for both the read only condition $\left[F(1,31)=7.54, M S_{\mathrm{e}}=2.64, p<.05, \eta_{\mathrm{p}}^{2}=.20\right]$ and the imagery condition $\left[F(1,31)=4.73, M S_{\mathrm{e}}=2.25, p<.05\right.$, $\left.\eta_{\mathrm{p}}^{2}=.13\right]$. There was no decline in performance whatsoever for the implementation intention condition $(F<1)$.

To further investigate the above finding, we tabulated the percentages of participants in each group whose prospective memory performance was attenuated by divided attention. In the control condition, $28 \%$ of the participants showed diminished performance due to divided attention; none performed better under divided attention. Similarly, in the imagery-only condition, 34\% of the participants performed worse under divided attention; $6 \%$ performed better under divided attention. In contrast, for the full implementation intention group, only $13 \%$ of the participants performed more poorly under divided attention; 9\% performed better under divided attention. These findings indicate that a majority of the participants in all the groups performed the prospective memory task equally well (or better) under standard and divided attention. Thus, the use of the implementation intention strategy appears to have primarily benefited the portion of the distribution represented by approximately one third of the participants, who otherwise would have experienced prospective memory failures under divided attention. We return to this point in the General Discussion, after first reporting performance on the ongoing activities.

RNG. Participants in the implementation intention group may have maintained high levels of prospective memory under high attentional demand by sacrificing performance on the RNG task. To examine this possibility, we evaluated participants' RNG sequences for the randomness of the digit sequences generated. As a comprehensive assessment, we computed three indexes of randomness: (1) redundancy; (2) the RNG score, which measures predictability of pair sequences (Evans, 1978); and (3) the equality of response alternative frequencies using the turning point index (Azouvi, Jokic, Van der Linden, Marlier, \& Bussel, 1996), which measures the number of responses that indicate a change between increasing and decreasing sequences. All three measures were computed using Towse and Neil's (1998) RGCalc program.

Table 2 displays the means of the three randomness measures for each instruction condition and for the no prospective memory control group. For each measure, a onefactor ANOVA was conducted. No significant differences were found among the four conditions for the redundancy measure $\left[F(3,124)=1.70, M S_{\mathrm{e}}=4.26\right]$, nor for the turning point index $(F<1)$. For the RNG score, although the means were fairly comparable across the conditions, they did differ significantly $\left[F(3,124)=3.76, M S_{\mathrm{e}}=.006\right.$, $\left.p<.05, \eta_{\mathrm{p}}^{2}=.08\right]$. Table 2 shows that the implementation intention group produced the lowest score (more random sequences), and the control group the highest score. Thus, the participants who used the implementation intention strategy clearly did not sacrifice performance on the secondary RNG task relative to participants who did not use implementation intentions, or in relation to participants who had no prospective memory task to perform.

Word rating task. We also evaluated performance on the word rating task in order to investigate whether the prospective memory conditions were associated with variations in the deployment of attention on the ongoing task and whether the prospective memory task significantly altered performance on the ongoing task. We computed the average proportions of words rated and the average response times in order to make a rating across all trials (see Table 3), excluding the four prospective memory trials and the corresponding trials for participants in the no prospective memory control. For both of the measures, an ANOVA was conducted with attentional demand as the within-participants factor and instruction condition as the between-participants factor. As expected, these analyses revealed a main effect of attentional demand on both proportion of items responded to $[F(3,124)=151.6$, $\left.M S_{\mathrm{e}}=.64, p<.05, \eta_{\mathrm{p}}^{2}=.55\right]$ and mean reaction time $\left[F(3,124)=224.9, M S_{\mathrm{e}}=34,825, p<.05, \eta_{\mathrm{p}}^{2}=.64\right]$. However, there was no effect of instruction condition on either proportion of items responded to $[F(3,124)=1.66]$ or mean reaction time $(F<1)$. There was no interaction between attentional demand and instructional condition for either reaction time $[F(3,124)<1]$ or proportion of items responded to $[F(3,124)=1.27]$. Thus, the implementation intention did not decrease the participants' focus on the ongoing task relative to the other prospective memory conditions, nor did the presence of a prospective memory task result in generally slower responding to the ongoing activity.

Table 2

Mean Performance on the Random Number Generation Task

\begin{tabular}{lccc}
\hline \multicolumn{1}{c}{ Instructional Group } & $\mathrm{R}$ & $\mathrm{RNG}$ & $\mathrm{TPI}$ \\
\hline Implementation intention & 2.07 & .29 & .92 \\
Imagery only & 2.85 & .32 & .92 \\
Read only & 2.53 & .31 & .94 \\
No PM control & 2.13 & .33 & .92 \\
\hline
\end{tabular}

Note-R, redundancy; RNG, Evans's (1978) random number generation index; TPI, Azouvi et al.'s (1996) turning point index; PM, prospective memory. 
Table 3

Mean Completion Rates and Response Latencies (RTs, in Milliseconds) on the Word Rating Task, As a Function of Instructional Group and Attention Demands, in Experiment 2

\begin{tabular}{lcccc}
\hline \multicolumn{1}{c}{ Instructional Group } & $\begin{array}{c}\text { Standard- } \\
\text { Demand } \\
\text { Response }\end{array}$ & $\begin{array}{c}\text { High- } \\
\text { Demand } \\
\text { Response }\end{array}$ & $\begin{array}{c}\text { Standard- } \\
\text { Demand } \\
\text { RT }\end{array}$ & $\begin{array}{c}\text { High- } \\
\text { Demand } \\
\text { RT }\end{array}$ \\
\hline Implementation intention & .99 & .90 & 2,240 & 2,610 \\
Imagery only & .98 & .90 & 2,280 & 2,611 \\
Read only & .98 & .88 & 2,239 & 2,585 \\
No PM control & .98 & .85 & 2,229 & 2,582 \\
\hline
\end{tabular}

Note-PM, prospective memory.

\section{GENERAL DISCUSSION}

In two experiments, implementation intentions produced better prospective memory performance than did either an imagery condition that did not incorporate the explicit verbal statement of the intention (when I see X, $I$ will do $Y$ ) or a no-imagery condition (Experiment 2). This is the first refereed study (Cohen \& Gollwitzer, 2008, was presented as an invited chapter) of which we are aware that has found significant benefits of implementation intentions with young adults on a laboratory prospective memory task. Extending the finding to young adults eliminates a possible boundary condition to implementation intention effects in prospective memory suggested by initial studies. Specifically, based on reports of implementation intention effects with older adults (Chasteen et al., 2001; Liu \& Park, 2004) and the absence of effects with healthy younger and middle-aged adults (Bennett et al., 2005; Kardiasmenos et al., 2004), one idea has been that the benefit of implementation intentions is limited to special populations, such as older adults (see Ellis \& Freeman, 2008). The present positive effects of implementation intentions on a laboratory prospective memory task with healthy young adults, along with the implementation intention effect found by Cohen and Gollwitzer, suggests that implementation intention effects in prospective memory are not limited to special populations with brain-related pathologies (e.g., those with multiple sclerosis; Kardiasmenos et al., 2004) or with presumed deterioration of attentional or cognitive resources (e.g., older adults).

Yet to be explained is why implementation intention encoding confers advantages to prospective memory. One idea is that requiring participants to state "When I see the word $\mathrm{X}$, I will do $\mathrm{Y}$ " could create a strong social commitment to perform the action, and for this reason, implementation intention participants could have assigned a higher degree of importance to the prospective memory task. Augmenting the importance of a prospective memory task can increase prospective memory performance, but typically does so at the expense of other ongoing activities (Einstein et al., 2005; Kliegel, Martin, McDaniel, \& Einstein, 2001, 2004). For our purposes, the implication is that participants with implementation intentions would have allocated controlled resources, or would have increased allocation in relation to the other conditions, to the prospective memory task, even in the presence of the demanding secondary task. As a consequence, the implementation intention participants would have been expected to sacrifice performance on the secondary task, the ongoing task, or both.

None of these expected decrements was obtained, however. For RNG, participants in the implementation intention group approximated randomness of the digit sequences about as well as did participants in the other prospective memory groups, and also as well as did the participants in the no prospective memory control (see Table 2). Similar findings were obtained for the proportion of word rating responses completed and for average reaction times (see Table 3). These performance levels on both the random number task and the ongoing word rating task disfavor the account that implementation intention instructions increased the importance of the prospective memory task (e.g., due to social commitment) in relation to the other instructional conditions.

Thus, the patterns just noted are not in line with a resource allocation shift toward the prospective memory task that is stimulated by the implementation intention. This implication and the finding that prospective memory performance with implementation intentions was not reduced under highly demanding, dual-task (RNG) conditionsconditions that produce decrements in prospective memory without implementation intentions - support the theoretical claim that implementation intentions create relatively reflexive processes for prospective remembering (Gollwitzer, 1999; Liu \& Park, 2004; Wilson \& Park, 2008). We hasten to add, however, that some aspects of the present results are not without interpretational ambiguities. In the demanding attention condition in Experiment 2, prospective memory performance under implementation intentions was nearly perfect, thereby reducing sensitivity to possible increases in prospective memory when implementation intentions were operative under standard attention demands.

Similarly, perhaps the presentation rate of the ongoing task was generous enough to mute sensitivity to any resource-demanding process incurred by implementation intentions. Inconsistent with this interpretation, using a very cost-sensitive ongoing task (lexical decision), Cohen and Gollwitzer (2008) reported that ongoing task responding was not significantly slowed when prospective memory was performed under implementation intention instructions, even though their prospective memory task was arguably more demanding than in the present case (three target events paired with three unassociated response words, as opposed to two target events paired with one keypress).

A further interpretational nuance regarding the present advantages of implementation intentions turns on whether implementation intentions supplanted effortful and controlled prospective memory processes, which might otherwise have been required under the standard prospective memory instructions, with automatic or reflexive responding (cf. Cohen \& Gollwitzer, 2008; Gollwitzer, 1999). An alternative possibility is that the present prospective memory paradigm already facilitated reliance on relatively reflexive prospective remembering, and implementation intention instructions amplified the likelihood that these reflexive processes would be operative.

Briefly, in the present paradigm, the encoded prospective memory target event, a comprehended word, was the focus 
of processing in the ongoing activity — comprehension and rating of semantic features of words. On some views of prospective memory, when the encoded target is focally processed during the ongoing activity, reflexive associative retrieval processes can contribute to retrieval of the intended action (McDaniel \& Einstein, 2007a, 2007b). However, the success of these reflexive retrieval processes depends on forging a strong associative link between the target event and the intended action (McDaniel et al., 2004; cf. Moscovitch, 1994). Under standard instructions, some, but not all, participants might successfully encode a strong associative linkage (cf. Kliegel et al., 2007), whereas implementation intention instructions would guide all or nearly all participants to form a strong associative link.

The patterns in Experiment 2 support the above theoretical analysis. A majority of participants in the control conditions maintained prospective memory performance under high-attention-demand conditions (RNG) at levels achieved without high attention demands (ongoing task only), and this prospective memory performance was accompanied by no decrement to either the ongoing task or the RNG task (relative to a no prospective memory control). This result suggests that under normal prospective memory instructions, many, but not all, participants spontaneously created strong associative links that gave rise to reflexive prospective memory responding (for a focal cue prospective memory task; Einstein et al., 2005). The increase in prospective memory performance levels for the implementation intention instruction condition was carried by an increase in the proportion of participants - in this case over $85 \%$ - who were not negatively impacted by high-attention-demand ongoing activity. The implication is that implementation intentions guided robust associative encoding that, for at least some participants would not have been stimulated through standard prospective memory instructions.

Although the present laboratory prospective memory task, using a small number of focal target events, may have fostered spontaneous associative retrieval processes (see Einstein et al., 2005), controlled or strategic processes appear necessary for prospective memory retrieval in other laboratory prospective memory tasks (Marsh \& Hicks, 1998; Smith, 2003). In these circumstances, implementation intentions might not improve prospective remembering. For instance, in some paradigms, the encoded target is not processed as part of the ongoing activity (e.g., the target event is a syllable, but the ongoing task emphasizes the processing of words), thereby requiring nonreflexive, strategic processes for prospective remembering, as indicated by costs to the ongoing activity (Einstein et al., 2005) and by robust declines in prospective memory when RNG is required (Marsh \& Hicks, 1998). In this type of paradigm, Breneiser (2007) found no hint of improved prospective memory with a range of imaginal and verbal implementation intentions.

Finally, the present patterns suggest that the implementation intention effect was not based entirely on imagery encoding, if at all. The effect was obtained in relation to a control condition that incorporated a parallel imagery encoding process (Experiments 1 and 2). If imagery were the functional component in the implementation intention effect, no differences in these conditions should have emerged. Furthermore, $30 \mathrm{sec}$ of imagining oneself performing the action in the presence of the prospective memory cue, without the accompanying verbalization of the condition-action statement, did not produce increases in prospective memory in relation to a control with no imagery (Experiment 2). Perhaps the particular kind of if . . then verbalization that is at the heart of Gollwitzer's (1999) conception of an implementation intention is needed to enhance associative encoding of the target event-intended action link (Cohen \& Gollwitzer, 2008; Kliegel et al., 2007; but see Breneiser, 2007, for other results), thereby producing performance advantages for implementation intention encoding strategies.

\section{AUTHOR NOTE}

We thank Nova Morrisette and Jeanine Sun for their assistance with data collection and analysis. Correspondence concerning this article should be addressed to M. A. McDaniel, Department of Psychology, Washington University, Campus Box 1125, St. Louis, MO 63130 (e-mail: mmcdanie(artsci.wustl.edu).

\section{REFERENCES}

Azouvi, P., Jokic, C., VAn der Linden, M., Marlier, N., \& BusSEL, B. (1996). Working memory and supervisory control after severe closed-head injury: A study of dual task performance and random generation. Journal of Clinical \& Experimental Neuropsychology, 18, 317-337.

Bennett, L. R., Clawson, D. M., \& Kardiasmenos, K. S. (2005, November). The retrospective component of prospective memory: Relationship to prospective component success. Poster presented at the 46th Annual Meeting of the Psychonomic Society, Toronto.

Brandstätter, V., Lengfelder, A., \& Gollwitzer, P. M. (2001). Implementation intentions and efficient action initiation. Journal of Personality \& Social Psychology, 81, 946-960.

Breneiser, J. (2007). Implementation intentions and cost in prospective memory retrieval. Unpublished doctoral dissertation, Washington University.

Chasteen, A. L., Park, D. C., \& Schwarz, N. (2001). Implementation intentions and facilitation of prospective memory. Psychological Science, 12, 457-461.

Cohen, A.-L., \& Gollwitzer, P. M. (2008). The cost of remembering to remember: Cognitive load and implementation intentions influence ongoing task performance. In M. Kliegel, M. A. McDaniel, \& G. O. Einstein (Eds.), Prospective memory: Cognitive, neuroscience, developmental, and applied perspectives (pp. 367-390). Mahwah, NJ: Erlbaum.

Cohen, A.-L., Jaudas, A., \& Gollwitzer, P. M. (2008). Number of cues influences the cost of remembering to remember. Memory \& Cognition, 36, 149-156.

CraiK, F. I. M. (1986). A functional account of age differences in memory. In F. Klix \& H. Hagendorf (Eds.), Human memory and cognitive capabilities: Mechanisms and performances (pp. 409-422). Amsterdam: Elsevier.

Craik, F. I. M., \& BYrd, M. (1982). Aging and cognitive deficits: The role of attentional resources. In F. I. M. Craik \& S. Trehub (Eds.), Aging and cognitive processes (pp. 191-211). New York: Plenum.

Einstein, G. O., McDaniel, M. A., Thomas, R., Mayfield, S., Shank, H., Morrisette, N., \& Breneiser, J. (2005). Multiple processes in prospective memory retrieval: Factors determining monitoring versus spontaneous retrieval. Journal of Experimental Psychology: General, 134, 327-342.

Einstein, G. O., McDaniel, M. A., Williford, C. L., Pagan, J. L., \& Dismukes, R. K. (2003). Forgetting of intentions in demanding situations is rapid. Journal of Experimental Psychology: Applied, 9, 147-162.

Ellis, J. A., \& Freeman, J. E. (2008). Ten years on: Realizing delayed 
intentions. In M. Kliegel, M. A. McDaniel, \& G. O. Einstein (Eds.), Prospective memory: Cognitive, neuroscience, developmental, and applied perspectives (pp. 1-27). Mahwah, NJ: Erlbaum.

Evans, F. J. (1978). Monitoring attention deployment by random number generation: An index to measure subjective randomness. Bulletin of the Psychonomic Society, 12, 35-38.

GollWITZER, P. M. (1999). Implementation intentions: Strong effects of simple plans. American Psychologist, 54, 493-503.

GuYNN, M. J. (2003). A two-process model of strategic monitoring in event-based prospective memory: Activation/retrieval mode and checking. International Journal of Psychology, 38, 245-256.

Kardiasmenos, K. S., Clawson, D. M., Wilken, J. A., \& Wallin, M. T. (2004, November). Effects of implementation intentions on prospective memory performance. Poster presented at the 45th Annual Meeting of the Psychonomic Society, Minneapolis, MN.

Kliegel, M., Martin, M., McDaniel, M. A., \& Einstein, G. O. (2001). Varying the importance of a prospective memory task: Differential effects across time- and event-based prospective memory. Memory, 9, 1-11.

Kliegel, M., Martin, M., McDaniel, M. A., \& Einstein, G. O. (2004). Importance effects on performance in event-based prospective memory tasks. Memory, 12, 553-561.

Kliegel, M., Martin, M., McDaniel, M. A., Einstein, G. O., \& Moor, C. (2007). Realizing complex delayed intentions in young and old adults: The role of planning aids. Memory \& Cognition, 35, 1735-1746.

KuHL, J. (1994). Action and state orientation: Psychometric properties of the action control scale (ACS-90). In J. Kuhl \& J. Beckmann (Eds.), Volition and personality: Action versus state orientation (pp. 47-59). Göttingen: Hogrefe.

LIU, L. L., \& PARK, D. C. (2004). Aging and medical adherence: The use of automatic processes to achieve effortful things. Psychology \& Aging, 19, 318-325.

MARSH, R. L., \& HicKs, J. L. (1998). Event-based prospective memory and executive control of working memory. Journal of Experimental Psychology: Learning, Memory, \& Cognition, 24, 336-349.

McDaniel, M. A., \& Einstein, G. O. (2000). Strategic and automatic processes in prospective memory retrieval: A multiprocess framework. Applied Cognitive Psychology, 14, S127-S144.

McDaniel, M. A., \& Einstein, G. O. (2007a). Prospective memory: An overview and synthesis of an emerging field. Thousand Oaks, CA: Sage.

McDaniel, M. A., \& Einstein, G. O. (2007b). Spontaneous retrieval in prospective memory. In J. S. Nairne (Ed.), The foundations of remembering: Essays in honor of Henry L. Roediger III (pp. 227-242). New York: Psychology Press.

McDaniel, M. A., Guynn, M. J., Einstein, G. O., \& Breneiser, J. (2004). Cue-focused and reflexive-associative processes in prospec- tive memory retrieval. Journal of Experimental Psychology: Learning, Memory, \& Cognition, 30, 605-614.

McDaniel, M. A., Robinson-Riegler, B., \& Einstein, G. O. (1998) Prospective remembering: Perceptually driven or conceptually driven processes? Memory \& Cognition, 26, 121-134.

Milne, S., Orbell, S., \& Sheeran, P. (2002). Combining motivational and volitional interventions to promote exercise participation: Protection motivation theory and implementation intentions. British Journal of Health Psychology, 7, 163-184.

Moscovitch, M. (1994). Memory and working with memory: Evaluation of a component process model and comparisons with other models. In D. L. Schacter \& E. Tulving (Eds.), Memory systems 1994 (pp. 269-310). Cambridge, MA: MIT Press.

MRC Psycholinguistic Database: Machine usable dictionary (Version 2.00). Retrieved February, 2001, from University of Western Australia: www.psy.uwa.edu.au/mrcdatabase/uwa_mrc.htm.

Orbell, S., Hodgkins, S., \& Sheeran, P. (1997). Implementation intentions and the theory of planned behavior. Personality \& Social Psychology Bulletin, 23, 945-954.

SheEran, P., \& Orbell, S. (1999). Augmenting the theory of planned behavior: Roles for anticipated regret and descriptive norms. Journal of Applied Social Psychology, 29, 2107-2142.

Smith, R. E. (2003). The cost of remembering to remember in eventbased prospective memory: Investigating the capacity demands of delayed intention performance. Journal of Experimental Psychology: Learning, Memory, \& Cognition, 29, 347-361.

Towse, J. N., \& NeIL, D. (1998). Analyzing human random generation behavior: A review of methods used and a computer program for describing performance. Behavior Research Methods, Instruments, \& Computers, 30, 583-591.

Turner, M. L., \& Engle, R. W. (1989). Is working memory capacity task dependent? Journal of Memory \& Language, 28, 127-154.

Wilson, E. A. H., \& PARK, D. (2008). Prospective memory and health behaviors: Context trumps cognition. In M. Kliegel, M. A. McDaniel, \& G. O. Einstein (Eds.), Prospective memory: Cognitive, neuroscience, developmental, and applied perspectives (pp. 391-410). Mahwah, NJ: Erlbaum.

\section{NOTE}

1. Note that the positive implementation intention findings cited at the outset contrasted the implementation intention condition with control conditions in which participants were not likely specifying particular situations in which to execute the action, such as "Take vitamin C."

(Manuscript received July 23, 2007; revision accepted for publication November 25, 2007.) 